\title{
Reflets
}

Revue ontaroise d'intervention sociale et communautaire

\section{Résistance et créativité : pratiques alternatives des familles démunies}

\section{Michèle Kérisit et Nérée St-Amand}

Volume 3, numéro 1, printemps 1997

Enfance et familles en contexte d'appauvrissement

URI : https://id.erudit.org/iderudit/026150ar

DOI : https://doi.org/10.7202/026150ar

Aller au sommaire du numéro

Éditeur(s)

Reflets : Revue ontaroise d'intervention sociale et communautaire

ISSN

1203-4576 (imprimé)

1712-8498 (numérique)

Découvrir la revue

Citer cet article

Kérisit, M. \& St-Amand, N. (1997). Résistance et créativité : pratiques alternatives des familles démunies. Reflets, 3(1), 30-54.

https://doi.org/10.7202/026150ar
Résumé de l'article

En utilisant des exemples concrets tirés de notre recherche sur les ressources alternatives pour familles démunies au Canada, nous illustrons comment de nombreuses familles défavorisées, avec l'aide d'organismes communautaires, résistent aux coups qui leur sont portés par une économie et un climat politique de plus en plus hostiles à leur qualité de vie. Les pratiques d’intervention décrites ici se centrent sur le développement des liens sociaux au sein des organismes eux-mêmes et au sein des communautés et par là même nous invitent à repenser les approches d'intervention possibles auprès des familles francophones démunies en Ontario.
Tous droits réservés (C) Reflets : Revue ontaroise d'intervention sociale et communautaire, 1997
Ce document est protégé par la loi sur le droit d'auteur. L'utilisation des services d’Érudit (y compris la reproduction) est assujettie à sa politique d'utilisation que vous pouvez consulter en ligne.

https://apropos.erudit.org/fr/usagers/politique-dutilisation/ 


\section{Résistance et créativité: pratiques alternatives des familles démunies}

En utilisant des exemples concrets tirés de notre recherche sur les ressources alternatives pour familles démunies au Canada, nous illustrons comment de nombreuses familles défavorisées, avec l'aide d'organismes communautaires, résistent aux coups qui leur sont portés par une économie et un climat politique de plus en plus hostiles à leur qualité de vie. Les pratiques d'intervention décrites ici se centrent sur le développement des liens sociaux au sein des organismes eux-mêmes et au sein des communautés et par là même nous invitent à repenser les approches d'intervention possibles auprès des familles francophones démunies en Ontario.

\section{Michèle Kérisit et Nérée St-Amand \\ École de service social, Université d'Ottawa}

À entendre les discours politiques actuels en Ontario, comme en Alberta, on serait porté à croire que les familles démunies et les personnes pauvres sont responsables de nos malaises économiques et sociaux (Harrison et Laxer 1995). On parle comme si dépendance, instabilité et manque de motivation sont des caractéristiques intrinsèques, presque naturelles, des personnes recevant l'assistance sociale. Pire encore, on les accuse de saigner à blanc les ressources financières de la province et d'être un fardeau trop lourd pour le contribuable moyen de l'Ontario. Ce discours dominant sur les familles défavorisées a une double conséquence. D'une part, il crée le sentiment, dans le reste de la population, que ces familles sont différentes, qu'elles constituent un groupe de personnes fondamentalement étrangères au mode de vie et à l'éthique des autres Ontariens (Ralph, Régimbald et St-Amand 1997). D'autre part, il justifie les mesures draconiennes qui réduisent constamment la marge de manoeuvre pourtant déjà 
réduite des familles défavorisées, alors que celles-ci ont très peu de moyens pour riposter, faire valoir leurs revendications et exiger leur dignité (De Gaulejac 1994; Berrick 1995).

Prenant à rebours ce discours dominant et victimisant, le présent article a pour but de mettre en valeur la créativité des familles démunies quand elles se mettent ensemble pour créer des solidarités dans leur milieu. À l'aide d'exemples concrets tirés de notre recherche sur les ressources alternatives pour familles démunies au Canada (St-Amand et al. 1996; Kérisit et St-Amand, 1994, 1995) ${ }^{1}$, nous tenterons d'illustrer comment des familles défavorisées, avec l'aide d'organismes communautaires, résistent aux coups que leur impose une économie de plus en plus insensible aux besoins du plus grand nombre. Nous tenterons également de soulever quelques pistes de réflexion sur la façon dont il faudrait actuellement repenser les pratiques professionnelles d'intervention auprès des familles défavorisées.

\section{Quelques enjeux de l'intervention auprès des familles défavorisées}

Nous n'avons pas l'intention ici de développer longuement les défis que pose la pauvreté au service social. Il nous faut cependant en repérer les grandes lignes afin de montrer combien les ressources communautaires que nous avons identifiées lors de notre recherche se démarquent des approches plus traditionnelles en vigueur dans de nombreuses agences et services d'aide à la famille.

\section{Qui est pauvre?}

Il existe de nombreuses études canadiennes sur la pauvreté. Généralement quantitatives, elles tentent de repérer les ménages qui n'ont pas de ressources matérielles suffisantes. Différents 
«Être pauvre, ce n'est donc pas, évidemment, choisir de l'être, comme le feraient peut-être croire les discours dominants dont nous avons parlé; c'est se trouver à la croisée de processus macro-économiques et structuraux.» indices de pauvreté sont alors utilisés (Mercier 1995). En traduisant la pauvreté en termes chiffrés, ces études statistiques, nécessaires à la gestion et à la planification de programmes gouvernementaux même minimums, nous donnent à voir une réalité relativement statique qui laisse peu de place à l'examen des mécanismes qui mènent à la pauvreté et à l'expérience même de la pauvreté. Or que constate-t-on?

D'une part, un examen des statistiques permet de constater que depuis quelques années, les visages de la pauvreté se sont grandement modifiés. Selon Ninacs, les configurations de la pauvreté se caractérisent par les traits suivants: «une incidence ciblée sur les jeunes familles, celles avec trois enfants ou plus, celles qui sont monoparentales [...], les personnes handicapées et les personnes ayant des problèmes de santé mentale [...]; une instabilité marquée par les revenus précaires d'emplois non standards, en particulier dans les villes; une dimension territoriale (appauvrissement des centres-villes et régions rurales au profit des banlieues); une intensité qui fait en sorte que les programmes d'assistance ne suffisent plus à couvrir les besoins vitaux des personnes concernées (1996:3-4).

Être pauvre, ce n'est donc pas, évidemment, choisir de l'être, comme le feraient peut-être croire les discours dominants dont nous avons parlé; c'est se trouver à la croisée de processus macroéconomiques et structuraux. Pour Deena White (1994: 42-43), «l'exclusion est à la fois un processus mondial et une expérience locale» qui touche, outre ceux que Ninacs nomme, les «travailleurs âgés et moins âgés perçus comme coûteux, lents ou incapables de s'adapter au changement», "les minorités visibles jugées non acculturées et perturbatrices de l'harmonie sur les lieux de travail», «les femmes chefs de famille qu'on suppose préoccupées et peu fiables ou qui sont censées préférer le travail à temps partiel malgré l'absence de sécurité et d'avantages sociaux». La pauvreté qui cible ces populations, nous l'avons retrouvée dans toute notre étude. Nos répondantes étaient en grande majorité des mères chef de famille monoparentale, c'est-à-dire l'une des grandes catégories ciblées par la pauvreté. 
"Jeter un regard

différent sur la

pauvreté, la voir à

travers les yeux et

l'expérience des

familles défavorisées et

prendre en

considération les

mécanismes de

l'appauvrissement

plutôt que l'état de

pauvreté nous obligent

donc à revoir les

approches que le

service social adopte

dans son aide aux

familles démunies.»
Ainsi observe-t-on qu'aux marginalités qui faisaient traditionnellement l'objet d'intervention en service social (sans abris, populations caractérisées par une déficience diagnostiquée, invalidité, troubles psychiques, etc.) s'ajoute à présent une marginalité beaucoup plus mouvante, non traditionnelle. Désignées comme "nouveaux pauvres», de nombreuses personnes viennent ainsi grossir les rangs des clientèles d'un système d'assistance sociale peu préparé à gérer des situations qui conjuguent misère matérielle, rupture des liens sociaux, communautaires, de travail, et familiaux. Castel (1994: 13) définit ainsi, entre une «zone d'insertion» combinant travail et relations familiales stables, et une zone de marginalité maximum (absence de travail et isolement relationnel), une «zone de vulnérabilité» caractérisée par le travail précaire et la fragilité des soutiens relationnels. Pour cet auteur, la véritable marginalité (la désaffiliation) survient à la fin d'un double parcours de décrochage vis-à-vis du travail et des relations, en particulier des relations familiales et de quartier. Ce qui caractérise notre société actuelle serait par conséquent, non pas tant l'augmentation absolue des zones de marginalité ou de désaffiliation que la croissance constante des «zones de vulnérabilité» où les gens vivent de «jobines» et dont les liens familiaux et sociaux sont particulièrement fragmentés et fragiles.

La planification propre aux actions de l'État en matière d'assistance sociale, en «fixant» dans les chiffres les besoins des populations, ne rend pas vraiment compte de ces processus et multiplie les angles sous lesquels la pauvreté est abordée (malnutrition, décrochage scolaire, santé mentale, poids insuffisant à la naissance, etc.), sans en voir l'unité structurelle. Selon Lesemann (1994), une telle "conception institutionnelle de la pauvreté» a pour conséquence également de concentrer l'attention sur l'individu comme seule unité d'enquête et d'intervention. On présume alors que l'individu est libre et "capable d'initiative et donc ultimement maître de ses choix» sans égard pour sa «qualité sociale, ses liens multiples, son insertion communautaire et son identité culturelle» (Lesemann 1994: 590).

Jeter un regard différent sur la pauvreté, la voir à travers les yeux et l'expérience des familles défavorisées et prendre en 
considération les mécanismes de l'appauvrissement plutôt que l'état de pauvreté nous obligent donc à revoir les approches que le service social adopte dans son aide aux familles démunies. En explicitant quelques exemples d'approches qui émergent en réponse aux besoins des familles défavorisées actuelles, nous espérons donner quelques raisons d'espérer aux intervenants et aux familles démunies qui se battent quotidiennement contre les conséquences de l'appauvrissement. C'est pourquoi un point majeur de notre recherche a été de repérer les groupes qui essaient non seulement d'alléger la misère économique mais aussi de créer ou recréer un sens communautaire et de retisser un tissu social effiloché par l'isolement. "Ce que nous essayons de faire dans notre programme, dit une personne interviewée, c'est, autant que possible, de créer une famille pour les gens isolés». Se battre contre la misère semble passer tout d'abord par la mise en place de nodules de protection comme les appelle l'une des intervenantes interrogées, nodules de protection suffisamment solides pour pouvoir ensuite confronter une société extrêmement hostile aux familles démunies.

\section{L'image de soi et la stigmatisation}

Dans la littérature traditionnelle du service social, les familles pauvres qui faisaient l'objet d'intervention par les travailleurs sociaux ont été désignées sous une série de vocables qui les dépeignaient tantôt comme unmotivated, resistant to offer of help (Meyer 1963), hard to reach (Tinker 1959), disengaged (Tomlinson and Peters 1981) ou tout simplement dysfonctionnelles. Ce sont, plus près de nous, les familles à problèmes multiples, à risque, les clientèles lourdes, désorganisées. Ces expressions mettent avant tout l'accent sur les déficiences. Les familles démunies posent problème, non nécessairement pour elles-mêmes (cela ne veut pas dire qu'elles n'en ont pas), mais certainement pour le professionnel dont le rôle est justement de régler le problème grâce à son expertise. C'est le regard que le professionnel porte sur la famille qui la définit. Ce regard professionnel a souvent 
"Ce regard

professionnel a souvent

pour effet de diviser et compartimenter à

l'infini les expertises alors que les difficultés ressenties par les clientèles défavorisées leur semblent appartenir à un tout interrelié.» pour effet de diviser et compartimenter à l'infini les expertises alors que les difficultés ressenties par les clientèles défavorisées leur semblent appartenir à un tout interrelié. Les programmes chargés de régler les conduites et d'apporter des changements en fonction d'apprentissages et de remodelages sur des comportements plus appropriés (cours d'habiletés parentales, de budgétisation de ressources en fait inexistantes, de préparation à des emplois qui n'existent pas) semblent alors voués à un certain échec. D'où la stigmatisation des familles que l'on veut rejoindre et la démoralisation de beaucoup d'intervenants qui ont de plus en plus de mal à régler des problèmes qu'il ressentent comme de plus en plus complexes (Lachapelle 1994).

Les familles pauvres ne se reconnaissent évidemment pas dans de tels vocables. "On est une ressource et non un fardeau pour la société. On refuse d'être traités comme un paquet de problèmes", dit l'une des mères rencontrées. "On n'est pas des problèmes, on est des gens», dit un autre parent interviewé. Ce refus d'être nommé par l'autre est à la fois significatif du fardeau que ressentent les personnes devant l'intervention et de leur conscience aigüe que cet étiquetage ne correspond pas à leur expérience. En fait,

Vivre dans la pauvreté, [...] c'est se percevoir à travers ce que les autres nous communiquent, c'est vivre avec l'image que l'on a de soi. C'est aussi avoir une conscience très nette de la société qui nous entoure, de ses structures et du rôle qu'on y joue... ou qu'on n'y joue pas. (Robichaud et al 1994: 18).

Le but de cet article est aussi de comprendre comment les familles pauvres que nous avons rencontrées et les ressources communautaires qui les aident sont à même de lutter contre cette stigmatisation et de mettre en oeuvre des stratégies de résistance au «buldozzage» dont elles sont victimes. La démocratie interne aux organismes étudiés et la façon de la mettre en oeuvre nous ont, en particulier, permis de voir les liens qui peuvent exister entre la mise en place des nodules de protection dont nous parlions plus haut et l'élaboration d'actes de résistance aux assauts d'une société de plus en plus individualisante et victimisante. Dans cette mesure, 
il nous semble que les stratégies de résistance mises en place par les organismes que nous avons étudiés concernent un public plus large que celui des familles démunies. Elles peuvent aussi être reprises par toutes celles et tous ceux qui font actuellement l'expérience des dislocations sociales et économiques et d'une souffrance qui va à la fois en deça et au-delà de la pénurie de bien-être matériel.

\section{La création de nodules de protection}

\section{Repenser l'espace et le temps}

«Pour moi, ici, c'est comme une couverture tissée. Tout marche ensemble et se tient. C'est ce que ça évoque chez moi: une couverture. C'est la sécurité et tout est tricoté serré», nous a dit une mère de famille d'un organisme de l'Ouest canadien qui a pour mandat d'engager les familles dans l'éducation et le développement des enfants. L'autre métaphore que les gens emploient pour décrire leur sentiment de sécurité et de chaleur est celle de la famille. "Je suis venue ici avec mes enfants et c'était comme une vraie famille. On se sent proche, très proche, et pourtant, on ne se connait pas», a remarqué une mère. Cette importance qu'on place sur un lieu sécuritaire ne doit pas être prise à la légère, puisque c'est en tissant des interactions sociales qu'elles construisent la base sur laquelle peut se réaliser le développement communautaire. Une organisatrice communautaire de Sudbury abonde dans le même sens:

Ce à quoi on fait face, c'est de travailler à créer une base communautaire où les gens vont se supporter les uns les autres, se comprendre les uns les autres et avoir des occasions de travailler ensemble de façon agréable. Ils ne pourront jamais faire front commun à moins de se comprendre et de se connaître comme des partenaires et des amis. Ce qui compte le plus, finalement, c'est de faire en sorte que les gens s'occupent des autres. 
"...temps et lieux

reposent sur des

valeurs culturelles

profondément ancrées,

façonnées par nos

coutumes et nos

comportements. Si les

ressources alternatives

$y$ attachent autant

d'importance, c'est que

respecter les besoins

des participantes, c'est

aussi respecter les

paysages et les

rythmes familiaux et

sociaux dans lesquels

la ressource est

enracinée. »
Les ressources que nous avons visitées essaient de créer un chezsoi. Ce faisant, elles font face à un double défi : d'une part il leur faut créer un lieu où il est possible de tisser des liens et de les renforcer, ce qui n'est pas chose facile dans un monde où l'idéologie dominante est basée sur la loi du plus fort. D'autre part, il leur faut s'assurer que leur situation financière soit assez solide pour lancer et maintenir des projets priorisant le mieuxêtre des participantes. Une telle démarche nécessite du temps, de l'engagement, de l'imagination pour penser autrement, faire autrement. C'est pourquoi nous avons appelé les ressources que nous avons contactées des ressources alternatives.

Dans un premier temps, le défi consiste donc à repenser et à réorganiser l'espace que les participantes aux programmes utilisent. Cet agencement de l'espace est indicatif de l'organisation du temps qui est vécu au sein même de la ressource. Ceci est particulièrement important dans la mesure où temps et lieux reposent sur des valeurs culturelles profondément ancrées, façonnées par nos coutumes et nos comportements. Si les ressources alternatives y attachent autant d'importance, c'est que respecter les besoins des participantes, c'est aussi respecter les paysages et les rythmes familiaux et sociaux dans lesquels la ressource est enracinée.

Les organismes visités n'organisent pas l'espace de la même manière que les services institutionnels, à la fois sur le plan de l'espace interne et dans ses relations spatiales avec la communauté environnante. À l'intérieur, la plupart des groupes que nous avons rencontrés s'étaient organisés autour d'une pièce commune, le plus souvent une cuisine, quelquefois une salle de réunion meublée d'un matériel un peu usé mais encore confortable. L'accueil et les rencontres ont presque toujours lieu dans cet espace commun qui incite à la discussion informelle. Pendant qu'on parle ou qu'on prend un café, on continue à travailler. Les enfants sont souvent présents ou bénéficient d'une pièce adjacente afin que mères et intervenantes puissent jeter un coup d'oeil sur leurs activités, même s'ils sont accompagnés d'une éducatrice. Ils ne sont donc pas considérés comme unité différente, un service différent. Au contraire, les enfants sont très souvent au coeur des ressources. "Ça commence là, quand t'es assise, tu prends un café, tu regardes 
dehors et puis tu vois les enfants. Les idées te marchent dans la tête pis c'est comme ça que ça commence. Puis on se dit: «on devrait faire ci, on devrait faire ça», nous a dit une participante.

Ce qui transpire d'une telle «culture informelle» (Hall, 1984: 228), c'est le goût des participantes d'avoir un espace ouvert, où les activités sont intégrées. Les priorités n'obéissent pas à une vision fonctionnelle de l'espace. L'effort du groupe se concentre sur la création d'une atmosphère, un cadre de vie privilégiant la chaleur humaine dans les liens entre les personnes. Certains organismes préfèrent par exemple repeindre leur local avant de réparer certaines parties structurales de l'édifice, ce qui prendrait plus de temps et d'argent sans pour autant créer des liens entre les gens. D'autres ont préféré mettre l'accent sur l'esprit communautaire en aménageant des plantes et des jardins collectifs.

D'autres organismes tentent de créer une autre forme d'espace d'interaction pour les personnes à faible revenu. C'est le cas, par exemple, de Field to Table de Toronto qui achète des produits frais des fermiers et d'autres fournisseurs et les livre aux groupes communautaires dans leur quartier. Cette ressource essaie ainsi de promouvoir une alimentation saine et économique, bien sûr, mais aussi de recréer un sens du marché, si important pour les nombreux Ontariens nouvellement arrivés de pays où la socialité passe en partie par le marché ouvert, lieu convivial par excellence.

Les programmes La boîte verte, qui connaissent une expansion remarquable depuis quelques mois, ont aussi le même objectif: on n'achète pas seulement de la nourriture au prix de gros, on crée des liens, on engage les gens, on bâtit des solidarités. Ce genre d'initiatives a pour but de donner aux communautés l'occasion de développer des liens interactifs et un sentiment d'appartenance. Alternatives aux banques alimentaires, ces formules permettent du même coup de régler, en partie du moins, les problèmes d'accès aux supermarchés, habituellement éloignés des quartiers et des logements à prix modique et de créer des espaces de solidarité et d'appartenance. L'acquisition de biens (matériels) passe par la création de liens et de l'échange (Godbout 1992). 
«Malgré les contraintes qu'impose souvent

l'architecture, les familles pauvres ont trouvé des façons originales d'organiser l'espace dont elles disposent et d'avoir des relations conviviales, plutôt que de simplement recopier les formes de pouvoir hiérarchiques.»
Réaménager l'espace implique une autre conception du pouvoir. Là où on a tenté de redéfinir l'espace dans lequel les participantes des organismes évoluent, on a changé du même coup la façon d'interagir, en redéfinissant les relations de pouvoir entre les gens et entre intervenants et familles. Comme une intervenante communautaire l'a remarqué, la disposition spatiale, dans les milieux institutionnels, intimide souvent les gens. «Les institutions, dit-elle, s'entourent d'un espace physique pour intimider les gens et pour renforcer le pouvoir de ceux qui ont du pouvoir sur les autres. Dans notre ressource, il y a beaucoup de créativité qui se manifeste, justement parce que les gens ont la liberté d'arranger l'espace à leur manière et le courage de ne pas reproduire le même type d'interactions que l'on retrouve ailleurs». Malgré les contraintes qu'impose souvent l'architecture, les familles pauvres ont trouvé des façons originales d'organiser l'espace dont elles disposent et d'avoir des relations conviviales, plutôt que de simplement recopier les formes de pouvoir hiérarchiques. Ce faisant, non seulement elles créent des nodules de protection marqués par le sentiment d'appartenance, de sécurité, le jeu et l'invention, mais elles résistent aussi, à leur façon et selon leurs moyens, aux forces idéologiques dominantes.

Temps et espace vont de pair. Les organismes alternatifs trouvent aussi des façons de contrer le problème de la mesure du temps que leur impose la culture dominante. L'un des commentaires les plus fréquents dans nos entrevues concerne le temps qu'il faut pour construire des rapports de confiance et donc une ressource qui marche. Plusieurs membres d'organismes que nous avons visités nous ont dit qu'ils appréciaient le fait que leur ressource n'était pas exclusivement à la merci d'un programme spécifique et spécialisé mais qu'il permettait surtout de "passer du temps ensemble». Ceci signifie alors que les activités organisées dans ces ressources n'obéissent pas nécessairement aux impératifs d'objectifs mesurables et mesurés en termes du temps que l'on met à obtenir un résultat. En décrivant son programme, une travailleuse communautaire nous a dit: «Notre programme n'est pas vraiment structuré. De cette façon, les gens peuvent venir quand ils le veulent durant la journée et ils ne sont jamais en 
"C'est autour de la table que l'accueil se fait, que les idées germent, que les échanges s'effectuent, que se créent des complicités.» retard pour rien. Ils n'ont donc rien à s'inquiéter car le programme est toujours là. Le Centre aide ses membres à bâtir des amitiés, des réseaux et des contacts».

Une conception différente du temps transparaît à travers cette remarque. Le temps est utilisé et mesuré dans un contexte d'interactions sociales. Le groupe fonctionne sur un mode polychronique (Hall, 1984: 58), c'est à dire qu'il utilise un rythme "où on peut faire plusieurs choses à la fois, où on n'est pas contraint à un horaire pré-établi». Le temps est centré sur les relations, sur le processus bien plus que sur les résultats ou la tâche. Ce temps de la création des liens sociaux correspond aussi à ce que Melucci (1989:104) appelle le «temps intime» constitué de moments subjectifs liés aux cycles naturels de la personne et à des expériences très personnelles sur le plan affectif et émotif. Ceci s'est manifesté clairement lors de nos visites : le café était toujours disponible; on prenait le temps de se parler, de rire. C'est autour de la table que l'accueil se fait, que les idées germent, que les échanges s'effectuent, que se créent des complicités.

Une organisation opérant selon un mode monochronique, par contre, "nous détermine subtilement à penser et à percevoir le monde de manière fragmentée; ceci permet de se concentrer sur une chose à la fois, mais se traduit aussi par un appauvrissement du contexte de la communication interindividuelle» (Hall 1984: 61-62). Cette dernière façon d'utiliser le temps est celle de la plupart des lieux de travail et des organisations bureaucratiques qui, justement, sont identifiées comme participant à l'exclusion des familles démunies. Melucci appelle une telle organisation du temps le «temps social». Elle est liée à une division objective du temps dictée par l'horloge. Le temps est fragmenté en particules consacrées à l'épicerie, au travail, aux rôles sociaux, etc.

L'une des commodités les plus rares pour les familles défavorisées ou vivant en situation de pauvreté est effectivement celle du temps: elles passent leur vie à se faire rappeler leurs obligations, à assumer avec peine et misère de nombreux rôles sociaux, à traiter avec bien des agences et des organismes, à attendre leur tour, sans compter l'usure quotidienne que leur imposent les 
soucis reliés à l'absence d'un moyen de transport (Burman,1988: 139). Selon nos observations, les ressources alternatives ont développé deux manières de s'organiser autour des deux pôles temporels définis plus haut. Certaines privilégient des pratiques où domine un temps polychronique et intime permettant aux gens de souffler et de se retrouver. Les différentes activités seront alors organisées toutes ensemble. «Tous les programmes ont lieu en même temps, nous a-t-on dit, la halte-garderie, la joujouthèque, les ateliers et les séminaires quand nous avons des invités. La garde des enfants a lieu en même temps». D'autres ressources se donnent pour mandat d'essayer de réconcilier temps intime et temps social; ce sont en général les organismes qui ont des programme axés sur la préparation à l'emploi. En laissant aux participantes la possibilité de retrouver un rythme de vie centré sur elles-mêmes, les ressources leur permettent de prendre un recul par rapport au temps social, et donc de mieux comprendre ses exigences dans le monde du travail.

La délation, par exemple, est devenue une pratique instituée en Ontario grâce aux fameuses snitch lines recevant des

dénonciations anonymes de supposés fraudeurs. Cette pratique a pour effet de mettre gravement en péril les réseaux informels déjà fragiles dans les projets d'habitation à prix modique et les autres endroits où les gens démunis se retrouvent en ghettos.
En établissant des règles différentes concernant le temps et l'espace, de nombreuses ressources nous montrent comment l'on peut créer des endroits de rencontre brisant l'isolement et la méfiance que les familles peuvent éprouver. Ceci est déjà une réusssite en soi puisque les contraintes structurelles de notre société, en Ontario comme ailleurs, ont tendance à empêcher les gens de former des rapports de confiance fructueux sur une base quotidienne. Nous constatons plutôt que la méfiance, quand on lui donne la chance, ne manque pas de s'installer. La délation, par exemple, est devenue une pratique instituée en Ontario grâce aux fameuses snitch lines recevant des dénonciations anonymes de supposés fraudeurs. Cette pratique a pour effet de mettre gravement en péril les réseaux informels déjà fragiles dans les projets d'habitation à prix modique et les autres endroits où les gens démunis se retrouvent en ghettos.

Par opposition, les ressources décrites ici créent en quelque sorte des lieux d'appartenance. Ici, c'est chez-nous, nous a-t-on souvent dit. L'on serait cependant en droit de se poser la question suivante: créer de tels espaces protégés suffit-il à faire en sorte que les familles puissent se prendre en charge et atteindre un 
niveau d'autonomie qui leur permette une réinsertion dans le monde social dominant? Il nous a semblé qu'une seconde dimension développée par les ressources alternatives contribuait à cette démarche de prise en charge. Cette dimension, on l'a retrouvée dans le développement de pratiques démocratiques et conscientisantes au sein même des organismes. Ce faisant, sont posés des actes de résistance aux pratiques généralement autoritaires dirigées contre les gens démunis. Les ressources deviennent des lieux où, comme le disait une intervenante, les gens généralement silencieux trouvent leur voix.

\section{Exigences de la démocratie}

"...ce qui compte, ce n'est pas seulement la structure formelle de l'organisme mais le processus qu'il met en branle pour faire fonctionner la démocratie et en faire une ressource utile pour les participantes."
Au départ de notre recherche, au moment du contact avec les organismes que nous nommons alternatifs, la participation démocratique des familles à tous les niveaux de décision de l'organisme nous semblait une exigence pour qu'ils soient inclus dans notre banque de données. Bien que nous n'ayons pas précisé les modalités d'une telle participation, il nous est paru graduellement évident que ces modalités étaient multiples, aussi différentes que les mandats des organismes eux-mêmes. Nous avons constaté cependant que nous prenions pour acquis que ce fonctionnement serait en place dès la naissance de l'organisme. En fait, la participation des familles s'est révélée être un processus permettant d'atteindre à un moment donné un certain niveau d'équilibre démocratique qui devait être constamment reconfiguré et modifié. Le processus de démocratisation lui-même, les questions qu'il soulève quant aux relations de pouvoir et d'efficacité pratique dans la gestion des organismes, semblent être d'une importance capitale pour que se révèle une culture de la résistance. En d'autres mots, ce qui compte, ce n'est pas seulement la structure formelle de l'organisme mais le processus qu'il met en branle pour faire fonctionner la démocratie et en faire une ressource utile pour les participantes. 


\section{Démocratiser l'organisme}

Plusieurs éléments contribuent à ce processus complexe. Comme l'ont fait remarquer plusieurs, il n'est pas facile de créer une structure qui permette aux personnes démunies de se faire entendre puis de transformer leur voix en action et en projets concrets. De nombreuses contraintes empêchent les familles

"Bien souvent, les discours sur la participation des gens démunis ne reconnaissent pas la nécessité du temps centré sur le processus et l'effort quotidien et répété pour réinventer de nouvelles formes de prise de décision.» démunies de mener à terme des projets de longue haleine. Bien souvent, les discours sur la participation des gens démunis ne reconnaissent pas la nécessité du temps centré sur le processus et l'effort quotidien et répété pour réinventer de nouvelles formes de prise de décision. Comme l'affirme une intervenante rencontrée:

Bien des gens, même s'ils ne travaillent pas formellement ou s'il ne suivent pas une formation, sont très occupés, juste pour subsister. Ceux qui ne font pas ça [travailler ou étudier] sont généralement déprimés et trouvent cela pénible de sortir et de faire des choses. Il y en a beaucoup qui sont au chômage ou qui reçoivent l'assistance sociale parce qu'ils ne sont pas en forme. Ils sont éprouvés par des maux de dos ou par la maladie. Ce sont toutes ces choses-là ensemble qui font que c'est difficile d'amener les gens à s'engager et à participer sur une base régulière.

Telle est, à notre avis, la raison majeure des échecs de démocratisation d'organismes pourtant bien intentionnés. Il est en effet plus facile de prendre les gens en charge, et il est difficile de se départir de cette habitude. De plus, comme le précisait une intervenante, «vous devez vous départir de votre pouvoir si vous voulez en donner aux autres... et cela fait peur à bien des gens». Enfin, la plupart des personnes qui organisent, mettent sur pied et voient au bon fonctionnement des ressources sont des femmes. Elles se sentent tiraillées entre leurs responsabilités familiales et la création de projets collectifs. Nous pourrions ainsi continuer à énumérer les nombreuses difficultés qui se dressent sur le chemin de la démocratisation. Regardons plutôt comment un organisme rencontré s'y est pris pour gérer son processus de démocratisation 
et quelles ont été les stratégies adoptées pour contourner les difficultés qui se sont présentées. La démarche suivante nous semble en effet illustrer celle de bien d'autres organismes.

Année 0 : Éclosion d'une idée.

L'idée de former un organisme nait quand deux travailleuses sociales et des animatrices communautaires (dont l'une était des Premières Nations et l'autre, d'un organisme de santé mentale) se rencontrent à l'occasion d'un atelier portant sur le développement communautaire. Elles se proposent alors de mettre sur pied un projet pour aider les familles démunies de la région.

Année 1: Recherche de financement et mise en marche du projet.

Un groupe de travailleuses sociales de première ligne provenant de sept agences différentes soumet au gouvernement une proposition de projet; la coordonnatrice actuelle fait partie de ce groupe fondateur. Par la suite, ce groupe se rencontre chaque semaine et développe une grande solidarité entre ses membres. Année 2 : Élargissement du projet.

Le projet est financé grâce à un programme de rechercheaction d'ampleur provinciale. Des leaders provenant des quartiers environnants caractérisés par la pauvreté de leurs résidants se joignent au groupe. On rédige les définitions de tâches devant servir à l'embauche des employées. Une fois le personnel embauché, le groupe établit un calendrier de rencontres hebdomadaires auxquelles participent le personnel ainsi que des membres de la communauté.

Trois ans plus tard: Formalisation de la structure et stabilisation. L'Association se forme et se dote d'une structure formelle. Elle étend son membership à toutes les participantes de l'organisme, ainsi qu'au personnel qui habite le quartier. Par contre, elle ne permet pas aux personnes qui habitent à l'extérieur du quartier d'accaparer plus de $25 \%$ du membership. L'Association tente ensuite de mettre sur pied une équipe gestionnaire de travail, mais l'initiative échoue. Elle forme des caucus établis en fonction des besoins prioritaires dans chaque communauté ethnique de la région. 
Elle nomme alors un conseil consultatif communautaire dont l'un des membres est un élu local, puis éventuellement, un conseil d'administration est élu, composé de deux représentants de chacun des quatre caucus à la base de l'Association (Autochtone, multiethnique, francophone et anglophone), de trois personnes représentant la communauté at large, et de deux intervenants travaillant dans l'organisme.

Cette description rapide d'une histoire parmi tant d'autres nous permet de dégager quelques points importants dans les processus de formation d'organismes visant la participation de ses membres.

- l'enracinement des structures de l'organisme dans la communauté géographique immédiate. Cela présuppose, évidemment, la présence de l'organisme dans le quartier défavorisé lui-même. En limitant la participation des membres extérieurs à la communauté, on limite ainsi la possibilité que la voix des participantes à la ressource soit noyée dans les discours de personnes qui, malgré leurs bonnes intentions, ne reflètent pas la composition ethnique ou de classe du quartier, comme cela arrive très souvent. On s'assure ainsi que les préoccupations des familles démunies seront bien celles qui ont voix au chapitre.

- le respect de la diversité du quartier. Elle est reflétée dans la structure organisationnelle de telle sorte que les familles puissent se reconnaitre dans les orientations de l'organisme.

- la prudence avec laquelle les organisatrices ont agi, ce qui évite trop d'échecs répétés et donc le découragement.

- le rôle important, surtout au départ, d'un groupe d'intervenantes qui non seulement se sont fortement impliquées dans le projet mais ont créé, entre elles, des liens de solidarité et d'amitié leur permettant de dépasser les intérêts individuels ou les agendas des organismes auxquels elles étaient rattachées. Cela signifie, bien sûr, non seulement qu'elles y ont bénévolement consacré du temps mais que leurs organismes employeurs leur ont laissé assez de flexibilité pour participer au projet. 
Une telle démarche ne peut cependant éviter certains écueils et peut être porteuse de fragilités qu'il faudra résoudre: l'harmonie entre les diverses communautés ethniques impliquées dans la gestion n'est pas garantie. C'est certainement l'un des points les plus délicats à résoudre dans un projet desservant une population aussi diversifiée, ce qui est le cas dans plusieurs régions de l'Ontario. Il est également possible que les intervenantes qui travaillent quotidiennement dans l'organisme se créent des façons de faire, de communiquer et répondent à des impératifs qui se démarquent des familles qui fréquentent l'organisme.

Afin d'éviter cela, cet organisme (comme d'autres que nous avons rencontrés), a développé des stratégies intéressantes. D'ailleurs, beaucoup d'organismes visités s'assurent dans leur politique d'embauche que les intervenantes aient une connaissance approfondie du milieu de vie des familles, soit parce qu'elles y habitent personnellement, soit parce qu'elles y ont vécu longtemps. L'un des critères d'embauche de tels organismes se résume au propos d'une coordinatrice d'un organisme de Vancouver: "They don't only talk the talk, they walk the walk». En d'autres mots, elles ne connaissent pas seulement le discours mais ont elles-mêmes vécu les difficultés des familles qu'elles vont aider. De nombreux organismes visités comptaient en effet dans leur personnel des femmes qui avaient été clientes du programme, puis qui avaient pris en charge l'un des aspects du programme, souvent de façon bénévole, pour ensuite être embauchées. Plusieurs sont éventuellement allées au collège ou même à l'université pour formaliser le savoir-faire qu'elles avaient acquis au sein de l'organisme et se donner davantage de flexibilité dans la recherche d'emploi.

Cette proximité des intervenantes et des familles qui fréquentent les organismes alternatifs s'inscrit, à notre avis, dans les processus de démocratisation des organismes en basant ces processus sur des mécanismes d'identification des intervenantes aux besoins des familles. «Les intervenantes du programme, nous a-t-on dit à Toronto, reflètent la diversité au sein de nos membres, soit parce qu'elles habitent dans le quartier, soit qu'elles aient l'expérience d'être réfugiées ou chef de famille ou encore parce qu'elles ont été elles-mêmes bénéficiaires de l'aide sociale.» 


\section{Ambiguïtés des emplois de proximité}

L'on peut donc dire que mettre sur pied des projets même s'ils sont de courte durée, fait en sorte que des personnes démunies, souvent des mères sans alliance, se donnent une formation et acquièrent des habiletés pour lesquelles elles recevront une rémunération. Une autre conséquence est que leur leadership communautaire aura été développé puis affirmé. En cela, les organismes alternatifs remettent en question un certain système d'aide sociale basé sur une expertise extérieure qui ne comprend pas ou qui comprend mal la culture, les dynamiques et l'histoire de la communauté. Dans plusieurs organismes on considère que ce que font les femmes participantes est un vrai travail, plutôt que du bénévolat. Les femmes reçoivent donc une rémunération et la ressource, dans un tel cas, devient un endroit permettant de faire des stages et d'acquérir une expérience conduisant à d'autres formes de revenus.

Bien qu'en surface de telles façons de faire pourraient rappeler le travail obligatoire, le workfare, il nous faut ici souligner l'énorme différence qui existe entre les mesures gouvernementales actuelles et cette stratégie d'implication des participantes dans des emplois de proximité. D'une part ce travail se fait sur une base volontaire, comme travail socialement utile. Ce n'est pas une forme voilée de travaux forcés que l'on exécute pour répondre aux conditions d'admissibilité à l'assistance sociale. D'autre part, la prise en charge est faite par les femmes elles-mêmes. Ce sont souvent elles qui établissent les priorités de leurs actions. En cela elles ne travaillent pas en fonction d'une "commande» d'organismes qui, pour faire face aux coupures financières actuellement imposées, utiliseraient à moindre coût une main-d'oeuvre à bon marché, peu importe sa motivation.

En s'organisant pour assurer la sécurité d'un quartier ou pour répondre à une demande collective de coopérative de traiteur (catering), comme nous l'avons vu dans certains organismes, elles contribuent à la qualité de vie de la collectivité. À plusieurs égards, 
«... on nous dit de former des entreprises pour supporter notre travail communautaire. Ce qu'ils omettent de dire, c'est que $80 \%$ de toutes les petites entreprises tombent à l'eau en dedans de cinq ans et que c'est un travail de 16 heures par jour pour ceux qui le font et presque sans salaire par dessus le marché.» les ressources communautaires occupent déjà une part importante de l'économie sociale (Quarter 1994; Ninacs 1992) ou de l'économie communautaire (Rosanvallon 1995).

Notons qu'une certaine ambiguité existe d'ailleurs dans la recherche d'un développement local qui ne serait pas fondé sur une recherche de la prise en charge collective (Shragge 1993; Perry 1994). Les discours gouvernementaux actuels pourraient laisser croire que ce qui est recherché, à travers la dévolution des pouvoirs aux collectivités et dans l'arrêt des subventions aux organismes communautaires, est une incitation à la débrouillardise locale, à l'émergence d'un leadership menant à la création de micro-entreprises. Un tel discours, extrêmement populaire en Ontario actuellement, ne tient évidemment pas compte du fait que, pour créer des emplois viables, il faut non seulement du temps, mais aussi du capital financier et surtout des compétences en gestion. Comme nous le disait une intervenante impliquée dans la mise sur pied de micro-entreprises, "on nous dit de former des entreprises pour supporter notre travail communautaire. Ce qu'ils omettent de dire, c'est que $80 \%$ de toutes les petites entreprises tombent à l'eau en dedans de cinq ans et que c'est un travail de 16 heures par jour pour ceux qui le font et presque sans salaire par dessus le marché». Ce sentiment de faire face à des défis insurmontables se vérifie dans la réalité : $80 \%$ de toutes les nouvelles petites entreprises ne franchissent pas le cap des 5 ans si on tient compte des entreprises de restauration ${ }^{2}$. En d'autres mots, vouloir démarrer une entreprise sans capital ni emprunt bancaire, sans connection et sans savoir-faire, est extrêmement difficile. Un tel développement local, fondé exclusivement sur le modèle de l'entreprenariat capitaliste, est au pire une illusion, au mieux, une entreprise de désinformation. De plus, alors que les coopératives mises sur pied par les ressources alternatives sont orientées avant tout sur le développement ou la réparation d'un tissu social extrêmement fragile, un modèle capitaliste de développement local a pour effet d'enfermer les gens dans une logique économique peu propice au souci des autres (Ninacs 1995).

Dans les ressources visitées, les modèles de développement sont très différents. Dans plusieurs communautés autochtones, 
par exemple, on développe l'art et l'artisanat, ce qui permet aux gens de renouer avec leur histoire et de gagner de l'argent lors de la vente d'objets utilisés lors de powwows traditionnels ou intercommunautaires. De telles activités sont susceptibles de soutenir les participants, de re-créer des liens sociaux et de développer un sentiment d'appartenance. Une coopérative de consommation tenue par les Premières Nations de Winnipeg nous indique:

Nous offrons des mocassins fabriqués à la main par des fermmes autochtones du quartier, des livres pour enfants autochtones, du pain bannock frais $d u$ jour et du café Bridgehead. Pour promouvoir la santé communautaire, nous offrons des fruits aux enfants à des prix d'enfants et nous ne vendons pas de cigarettes. C'est un investissement dans le partage et la dignité.

Il devient vite évident que pour bien des organismes alternatifs la recherche d'un profit n'est pas la préoccupation principale, souvent parce qu'il est impossible à réaliser surtout dans les premières années. Les quartiers que nous avons visités, comme celui où est installé la coopérative que nous venons de citer, reviennent de loin et ont encore besoin que l'État fournisse une aide financière appropriée pour soutenir les initiatives de développement local.

\section{Pertinence et limites des ressources alternatives}

Dans cette dernière section, nous voudrions mettre en lumière la pertinence des solutions qui ont été développées par certaines associations ou organismes tant pour faire face aux coupures actuelles en Ontario que pour le développement des communautés franco-ontariennes. Nous poserons également la question des limites de ce genre d'intervention.

Ré-inventer un espace de vie pour faire face à l'appauvrissement des familles prend du temps et de l'argent. Plusieurs services essentiels ont été réduits ou éliminés et les bénéfices de l'aide sociale générale ont été réduits de $22 \%$. Comme nous l'avons vu, le gouvernement ontarien actuel propose des solutions 
de développement local qui, si elles ne sont pas soutenues longtemps et fermement grâce à l'argent public, n'auront pas, c'est le moins qu'on puisse dire, les effets escomptés. De plus, les autres solutions qui concernent le bénévolat ne sont pas à notre avis, de véritables solutions car très éloignées de possibilités d'empowerment collectif.

À titre d'exemple, voici comment un ministre ontarien conçoit le travail communautaire :

Les voisins qui aident les personnes âgées à domicile, les corporations qui parrainent des programmes de nutrition pour les enfants, les clubs sociaux qui financent des projets communautaires, les employés et les cadres du secteur privé qui font du bénévolat en procurant des services publics, tel est notre sens des valeurs en Ontario. Votre gouvernement est prêt à appuyer et encourager ces initiatives. (Globe and Mail, 29 septembre 1995, A12).

Un tel retour à une charité privée, telle qu'on la connaissait avant la mise en place de l'État-providence, prône la substitution de l'intérêt collectif par l'intérêt privé. Il est évidemment en droite ligne d'un retour aux valeurs conservatrices nord-américaines pour lesquelles l'individu est sommé de réussir et blâmé pour ses échecs. Cependant, ce qui nous frappe, ce n'est pas tant la désuétude et l'orientation victimisante que peut prendre une démarche de soutien du bénévolat (où la personne pauvre ou mal prise dépend $\mathrm{du}$ bon vouloir aléatoire et souvent condescendant des autres), c'est surtout l'impossibilité, dans notre monde, de mettre en marche de telles actions. Dans une société marquée de plus en plus par la compétition au niveau de l'emploi, par un accroissement des obligations familiales et de travail, pour ceux qui en ont, il y a lieu de se demander si des employés et des cadres du secteur privé auraient même le temps de faire du bénévolat. On peut se demander ce qui se produirait chez les personnes âgées en perte d'autonomie si on envoyait chez elles ces bonnes voisines (Lessing 1985) pour s'occuper d'elles, quand on sait à quel point les aînés tiennent à leur indépendance. Nos lecteurs savent enfin 
"Un développement local soutenu et important et le développement de leaderships communautaires ne peuvent se faire sans le soutien de l'État, qui est chargé du bien-être de tous ses citoyens. En essayant de faire revivre une époque révolue, où les riches s'occupaient des pauvres, en coupant le minimum vital aux familles démunies, en refusant un appui financier continu aux organismes communautaires et en mettant l'accent sur l'efficacité mesurable et technocratique des actes d'intervention, les politiques actuelles vont en fait à l'encontre de ce qu'elles semblent prôner, c'est à dire l'autonomie et la prise en charge." combien il est difficile de délier les cordons de la bourse d'entreprises et de corporations qui invoquent justement le contexte compétitif que leur impose l'économie actuelle pour ne rien donner. Un développement local soutenu et important et le développement de leaderships communautaires ne peuvent se faire sans le soutien de l'État, qui est chargé du bien-être de tous ses citoyens. En essayant de faire revivre une époque révolue, où les riches s'occupaient des pauvres, en coupant le minimum vital aux familles démunies, en refusant un appui financier continu aux organismes communautaires et en mettant l'accent sur l'efficacité mesurable et technocratique des actes d'intervention, les politiques actuelles vont en fait à l'encontre de ce qu'elles semblent prôner, c'est à dire l'autonomie et la prise en charge. Il n'y a pas plus sûr moyen pour démobiliser les communautés et détruire les initiatives des familles pauvres qui existent ici et maintenant. La prise en charge et l'autonomie ne peuvent exister que si certaines solidarités du milieu ont été mises en place ou préservées.

Les organismes communautaires franco-ontariens qui se sont développés dans les vingt dernières années se trouvent confrontés aux mêmes difficultés que tous les organismes que nous avons rencontrés: manque de ressources financières, démotivation des gens de la communauté qui manquent de temps et d'argent, action exclusivement orientée sur la survie pure et simple, que ce soit au niveau individuel ou collectif.

Cependant, le discours dominant actuel sur la prise en charge locale pourrait paraitre attrayant car il semble, en surface, promouvoir les initiatives locales et pourrait donc offrir un certain espace pour la prise en compte des particularités francophones de la province. Par contre, il faudrait se souvenir que c'est à la conjonction d'une intervention de l'État (sous forme de subventions, de concertation et de législation) et des initiatives de base que les communautés ont pu mettre en place les quelques services disponibles aux familles démunies. Les risques de la décentralisation des pouvoirs au niveau local sont grands, car ils pourraient signifier non seulement la perte des quelques bénéfices que pouvait apporter la Loi 8 sur les services en français ${ }^{3}$ dans le 
domaine des services aux familles démunies mais surtout signaler un repli sur des considérations de survie matérielle. Cette préoccupation, centrée exclusivement sur les besoins dits de base, serait alors coupée du cadre culturel et social sans lequel ne peuvent se développer les liens sociaux nécessaires, justement, à un véritable développement.

Que pourrions-nous faire, alors, en ces temps de vaches maigres? Nous avons tenté de montrer qu'il était possible de faire les choses différemment selon l'utilisation de l'espace, du temps et des rapports interpersonnels internes à un organisme. Ces façons de faire laissent entrevoir des gestes créatifs de résistance au tout individuel, au tout compétitif, au tout charité. En centrant notre intervention sur le développement de nodules de protection où les familles démunies peuvent se retrouver, malgré la pauvreté des organismes, nous créons les conditions nécessaires au positionnement d'actes de résistance qui bénéficieront aux familles démunies, bien sûr, mais aussi à l'ensemble de la collectivité.

\section{Bibliographie}

BERRICK, J.D. (1995). Portraits of Women and Children on Welfare. Faces of Poverty, New York, Oxford University Press.

BURMAN, P. (1988). Killing Time, Losing Ground: Experiences of Unemployment, Toronto, Thompson Educational Publishing.

CASTEL, R. (1994). «La dynamique des processus de marginalisation: de la vulnérabilité à la désaffiliation", Cahiers de recherche sociologique, no 22,12-27.

DE GAULEJAC,V. et I.T.LEONETTI (1994). La lutte des places, Insertion et désinsertion, Paris, éditions Épi.

GODBOUT, J.T. (1992). L'esprit du don, Paris, Éditions La découverte.

HALL, E. (1984). La danse de la vie, Paris, Seuil.

HARRISON, T.et G. LAXER. (1995). The Trojan Horse. Alberta and the Future of Canada, Montréal, Black Rose Books.

KÉRISIT M. et N. ST-AMAND (1995). «Taking Risks with Families at Risk», dans J. Hudson et B. Galaway, éd. Child Welfare in Canada, Toronto, Thompson Educational Publications, 154-168.

KÉRISIT, M. et N. ST-AMAND (1994). «Familles-problèmes ou familles ressources. Approches alternatives auprès des familles démunies» dans G. Pronovost, éd.Comprendre la famille, Ste-Foy, Presses de l'Université du Québec, 31-49.

LACHAPELLE, R. (1994). «Une approche par clientèle ou une approche par milieu?» dans L. Chagnon, L. Favreau et R. Lachapelle, Pratiques d'action communautaire en CLSC: acquis et défis, Sainte Foy: Presses de l'Université du Québec, 87-97. 
LESEMANN, F. (1994). «La pauvreté: aspects sociaux», dans F. Dumond, S. Langlois et Y. Martin, éd. Traité des problèmes sociaux, Québec: Institut québécois de recherche sur la culture, 581-604.

LESSING, D. (1985). Le journal d'une bonne voisine. Les carnets de Jane Sumers, Paris, éditions Albin Michel.

MELUCCI, A. (1989). Nomads of the Present. Social Movements and Individual Needs in Contemporary Society, London, Brookmount House.

MER CIER, L. (1995). «La pauvreté: phénomène complexe et

multidimensionnel», Service social, vol. 44, no 3, 7-28.

MEYER, C. (1963). «Individualizing the Multiproblem Family», Social Casework, vol. 44 no 5: 267272.

MINISTÈRE DE LA SANTÉ DE L'ONTARIO (nd). La Loi sur les services en français 1986. Guide pour la désignation des agences. Toronto, Gouvernement de l'Ontario.

NINACS, W. A. (1996). Le service social et la pauvreté. De la redistribution des ressources à leur contrôle? Québec: Série Essais et synthèses, Laboratoire de recherche, École de service social, Université Laval.

NINACS, W. A. 1995. «Entraide économique, création d'entreprises, politiques sociales», Nouvelles pratiques sociales, vol. 8 , no 1,97-119.

NINACS,W. 1992. «Le mouvement populaire et communautaire dans le développement de l'économie sociale québécoise», dans N. Boucher, éd. Développement local et développement social: acteurs, institutions, enjeux, politiques. Tome 1: Développement local, Actes du 3ème séminaire interdisciplinaire international, Laboratoire de recherche en service social, École de service social, Université Laval, 161-174.

PERRY, S. et M. LEWIS (1994). Reinventing the Local Economy, Vernon, Centre for Community Enterprise.

QUARTER, J. (1994). Canada's Social Economy, Toronto, James Lorimer \& Co.

RALPH, D., A. RÉGIMBALD et N. ST-AMAND (1997). Open for Business, Closed to People. Mike Harris' Ontario, Halifax, Fernwood Press.

ROBICHAUD, J.-B., L. GUAY, C. COLIN et M. POTHIER (1994). Les liens entre la pauvreté et la santé mentale, Montréal, Gaëtan Morin éditeur.

ROSANVALLON, P. 1995. La nouvelle question sociale: repenser l'État-providence, Paris, éditions du Seuil.

ST-AMAND N., M. KÉRISIT, L. MARTINEAU, G. CLOUTIER, B. MALENFANT. 1996. This is our place / Ici c'est chez nous, Ottawa: Université d'Ottawa.

SHRAGGE, E. 1993. Community Economic Development: in Search of Empowerment, Montréal, Black Rose Books.

TINKER, K. H. 1959. «Casework with Hard-To-Reach Families», American Journal of Orthopsychiatry, vol. 29, no 1,165-171.

TOMLINSON, R et P.PETERS (1981). «An Alternative to Placing Children:Intensive and Extensive Therapy with Disengaged Families» Child Welfare, vol. 60, no 2, 95-103.

WHITE, D. (1994). «La gestion communautaire de l'exclusion», Lien social et Politiques - RIAC, no $32,37-52$. 
1. Pour un compte-rendu de la méthodologie et des résultats de cette recherche, voir St-Amand et al. Familles pauvres, alternatives aux interventions actuelles, Rapport final de recherche, École de service social, Université d'Ottawa (1994) et Ici, c'est chez nous / This is Our Place, Alternative resources for Low-Income Families / Ressources alternatives pour familles démunies, édition révisée, janvier 1996. Ce dernier ouvrage est un répertoire comprenant plus de 350 entrées décrivant le travail de quelque 300 ressources alternatives et de leurs regroupements provinciaux. La plupart des exemples évoqués dans cet article sont tirés de ce répertoire qui est disponible auprès des auteurs.

La recherche, menée en 1993 et 1994, a bénéficié du soutien financier du ministère du Développement des ressources humaines, Canada. Elle a été possible grâce à l'accueil, la collaboration et la générosité de nombreuses intervenantes et intervenants et de nombreuses mères de famille qui continuent d'inventer des pratiques originales d'entraide et de solidarité dans un contexte de plus en plus difficile. Notre travail leur est dédié.

2. Ces taux d'échec baissent à $45 \%$ si l'on exclut les entreprises de restauration (Ninacs, W. : communication personnelle). Ce dernier chiffre de $45 \%$ constitue tout de même un taux d'échec très élevé.

3. Adoptée en 1986, la Loi sur les services en français de l'Ontario (Loi 8) assure aux Ontariens francophones le droit de recevoir des services gouvernementaux en français dans 23 régions désignées où le nombre de francophones est d'au moins 5000 , ou représente $10 \%$ de la population. Les agences de paiement de transfert ne sont pas obligées par la législation d'offrir des services en français bien qu'elles soient invitées à le faire sur une base volontaire. Les municipalités sont exclues de la législation et ne sont pas tenues par la Loi à offrir des services en français, même si elles sont aussi invitées à le faire (Ministère de la Santé de l'Ontario, nd). 